\title{
Estudio de las interacciones profesor-alumno en la alfabetización de la geografía
}

Study of Teacher-Student Interactions in Geo-Literacy

Estudo das interações professor-aluno na alfabetização da geografia

\author{
Elsa Patricia Molina-Valdés* (iD orcid.org/0000-0002-3168-3233 \\ Valentina Haas-Prieto** (iD) orcid.org/0000-0001-9511-2766
}

\begin{abstract}
Artículo de investigación
Revista Colombiana de Educación, N. 75. Segundo semestre de 2018, Bogotá, Colombia.

doi: 10.17227/rce.num75-8101

Para citar este artículo: Molina-Valdés, E. y Haas-Prieto, V. (2O18). Estudio de las interacciones profesor-alumno en la alfabetización de la geografía. Revista Colombiana de Educación, 75, 59-76.
\end{abstract}

Profesora básica con mención en Lenguaje y Comunicación de la Pontificia Universidad Católica de Valparaíso. Docente Escuela Municipal de la comuna de Valparaíso. Correo electrónico: elsa.molina.v(a)gmail.com

** Doctora en Educación, mención Currículo y Didáctica. Coordinadora de prácticas de la Carrera de Pedagogía en Educación Básica de la Pontificia Universidad Católica de Valparaíso 


\section{Resumen}

En este artículo de investigación se describen las interacciones de los docentes en la alfabetización de geografía en una escuela municipal de Viña del Mar (Chile). Para ello y desde un enfoque cualitativo y mediante un estudio de caso instrumental, se analizan dos unidades curriculares de tercero y sexto básico, segmentando la interacción en diferentes unidades de análisis (Sánchez, García y Rosales, 2010). Los hallazgos dan cuenta de que los intercambios comunicativos no se condicen con las formas de alfabetizar de la disciplina, en cuanto al desarrollo de contenidos, habilidades y actitudes específicas de cada área del saber según lo que se busca favorecer en los cursos y niveles escolares estudiados.

\section{Palabras clave}

Interacción; unidades curriculares; intercambios comunicativos; Alfabetización en Geografía

\section{Keywords}

Interaction; curricular units; communicative exchanges; Geo-Literacy
In this research article we describe the interactions of teachers in geo-literacy in a municipal school in Viña del Mar (Chile). For that purpose, and from a qualitative approach and through an instrumental case study, two curricular units of the third and sixth grade are analyzed, segmenting the interaction in different units of analysis (Sánchez, García $\&$ Rosales, 2010). The findings show that communicative exchanges are not related to the literacy of the discipline, in terms of the development of content, skills and attitudes specific to each area of knowledge according to what is sought to favor the courses and school levels studied.

\section{Resumo}

Neste artigo de pesquisa descrevem-se as interações dos professores no ensino da Geografia em uma escola municipal de Viña del Mar (Chile). Para isso e desde uma abordagem qualitativa, por meio do estudo de caso instrumental, analisam-se duas unidades curriculares de terceiro e sexto ano do ensino fundamental, segmentando a interação em diversas unidades de análise (Sánchez, García e Rosales, 2010). As descobertas evidenciam que os intercâmbios comunicativos não concordam com as formas de ministrar a disciplina, em relação com o desenvolvimento de conteúdos, habilidades e atitudes específicas de cada área do conhecimento segundo os objetivos dos cursos e níveis escolares estudados.

\section{Palavras-chave}

interação; unidades curriculares; intercâmbios comunicativos; alfabetização em Geografia 


\section{Introducción}

El estudio de la interacción en el aula está marcado por dos cambios que suponen una ruptura en los principios básicos de la investigación educativa (Coll, 2010). El primero se relaciona con la creciente aceptación de los enfoques socioculturales y situados de la cognición, el aprendizaje y la enseñanza. Y el segundo, con la importancia paulatina otorgada al contexto en el aula.

Las interacciones en el aula entre docente y estudiantes permiten la internalización de conductas sociales y de procesos cognitivos superiores, los cuales son objetivos primordiales de la educación además de favorecer, comunicar, negociar significados, ayudando a construir representaciones y a desarrollar los propios sistemas de significados compartidos.

Ahora bien, desde la semiótica social, la creación de significado está guiada por modelos culturales que les indican a los usuarios los recursos que se deben utilizar y cómo ponerlos en juego en cada ámbito de actividad humana (Bajtín, 2002); formas que están situadas y legitimadas social y culturalmente. Del mismo modo, las asignaturas constituyen contextos específicos sobre una manera particular de ver el mundo (Halliday, 1982), incidiendo en formas de comunicar y representar, determinando el tipo de interacciones verbales que debieran propiciarse para contribuir al logro de sus propios objetivos.

Los resultados de la Evaluación del Desempeño Profesional Docente en Chile ${ }^{1}$ indican que solo el $18,1 \%$ de los docentes es competente en el criterio de interacción pedagógica. Este evalúa su capacidad para establecer interacciones que permitan el desarrollo cognitivo del pensamiento de los estudiantes a través de la calidad de las "preguntas planteadas, explicaciones desarrolladas, retroalimentaciones y las habilidades propias del subsector que enseña" (Flores y Abarzúa, 2013, p. 49).

Desde la certeza que las interacciones profesor/alumno son claves para comprender los procesos pedagógicos que subyacen a los resultados de aprendizaje de los estudiantes en cada área del saber (Galton, Hargreaves, Comber, Wall y Pell, 1999), en esta investigación se pretende responder la pregunta: ¿Cómo son las interacciones que propician los docentes con sus estudiantes en la alfabetización de geografía en un tercero y sexto básico de un Colegio Municipal de la comuna de Viña del Mar?

1 Entregados por el Centro de Perfeccionamiento, Experimentación e Investigación Pedagógicas CEIP, (2011) y la Subsecretaría de Educación Chilena. 


\section{Marco teórico}

\section{Interacción y aprendizaje en el aula}

Hay consenso en que la interacción dentro del aula es una de las influencias más importantes en el aprendizaje del estudiante (Wells, 2001) aunado al aporte del enfoque sociocultural que defiende el papel del mundo social y el contexto en el desarrollo cognitivo del sujeto (Vygotsky, 1988). Se considera así que "los procesos psicológicos superiores tienen su origen en la vida social, en las interacciones con otras personas, en la participación en actividades reguladas culturalmente" (Cubero et al., 2005, p. 50); en las que además se "adquieren destrezas y formas de conocimiento socioculturalmente valoradas" (Mead, 1934, p. 134). De ahí que la sala de clases sea concebida como un espacio donde "los hechos objetivos de la enseñanza y los aprendizajes [...] se constituyen y se definen a través de la interacción entre profesores y alumnos" (Mehan, 1979, p. 72).

En este sentido, Vygotsky (1988), a través del concepto de mediación semiótica, se refiere a los recursos como herramientas psicológicas que poseen un

[...] carácter social, ya que, por una parte, son producto de una práctica social y el acceso del individuo a ellos está asegurado por su pertenencia a un medio sociocultural y, por otra, son adquiridos a través de la interacción cara a cara. (Benítez, 1998, p. 367).

Esto se condice con la teoría de la semiótica social, en referencia al estudio de los recursos mediante los cuales los seres humanos favorecen la comunicación y transmisión de la cultura (Oteíza, 2009).

Halliday y Martín (1993) plantean que el lenguaje es un recurso para significar al servicio de las necesidades de los grupos sociales, enfatizando que la lengua es un "sistema de signos cuyo fin es el ser utilizado para crear significados con el propósito de intercambiarlos" (Abril, 1995, p.35) y desempeña la función más importante en el desarrollo de las personas como seres sociales.

Esta nueva mirada da cuenta que el estudio del desarrollo cognitivo se ha aproximado progresivamente a los aspectos específicos de la actividad cognitiva, situando al contexto en una posición mucho más importante. Así, según Rogoff (1998) se desprende que "los niños adquieren destrezas que les permitan abordar problemas culturalmente definidos, con la ayuda de instrumentos a los que fácilmente pueden acceder, y que construyen, a partir de lo que han recibido en el contexto de la actividad sociocultural" (p. 30). En consecuencia, la interacción en el aula se caracteriza por darse 
en un contexto específico, que favorece la construcción de significados compartidos y que responde a normas sutiles pero efectivas que organizan y regulan los papeles de cada participante.

Por tanto, concebiremos la interacción como el "conjunto de intercambios comunicativos o turnos discursivos que tienen lugar durante el desarrollo de las tareas académicas que integran la vida en el aula" (Sánchez, García y Rosales, 2010). Además de asumir que la interacción entre profesores y estudiantes debe analizarse en su contexto y dentro de la totalidad de la estructura de la actividad curricular (Christie, 2002).

\section{El proceso de alfabetización en las salas de clases}

Actualmente se concibe la alfabetización como un proceso de formación social y cultural, propiciado a través de la comunicación y los conocimientos que se construyen, por medio de las interacciones con otras personas (Heath, 1983), las que además van facilitando el aprendizaje y la enseñanza (Kress, 2010).

Asimismo, se reconoce que no existe una sola manera de alfabetizar, sino que hay diversas formas de lograrlo, por medio de un conjunto de prácticas comunicativas que se enseñan a lo largo del proceso académico, las cuales se denominan alfabetizaciones múltiples (New London Group, 1996). Ello, dada la diversidad de medios que van mucho más allá de lo escrito, abarcando títulos, tamaños, imágenes, sonidos. Lo anterior se sustenta en la heterogeneidad de las disciplinas que, al provenir de campos epistemológicos diferentes (Lemke, 1998), implican variadas visiones de mundo, privilegiando las interacciones que determinan ciertos aprendizajes (Álvarez y Del Río, 2003; Lemke, 1998). Dicho de otra forma, cada disciplina busca desarrollar en los estudiantes nociones, conocimientos, habilidades y actitudes distintas, esenciales para que el sujeto que aprende sea capaz de entender los fundamentos y características propias de cada asignatura (Bernstein, 1990) y con ello una determinada forma de construir conocimiento, junto a una especial visión de mundo. Sin embargo, uno de los errores más frecuentes, cometidos por los profesores en el aula, es que no precisan el discurso de la disciplina que enseñan, dado que no utilizan un lenguaje específico y coherente con los propósitos de cada área del saber (Manghi, 2013) y con ello la alfabetización no alcanza todo su potencial ni desarrolla lo esperado.

\section{La alfabetización en geografía}

Ante la conceptualización de geografía, lo primero es establecer que no hay consenso definitivo entre los especialistas. Mientras que para unos es una síntesis espacial, diferente y singular (Arenas y Salinas, 2013; Moreira, 
2010), para otros constituye un puente entre las ciencias naturales y sociales (Arenas y Araya, 2017); ante lo cual, la dicotomía entre geografía física y geografía humana debería diluirse.

Para efectos de este trabajo, la geografía se considera una ciencia social que aborda el estudio del suelo, la organización y estructura espacial de las sociedades humanas, las influencias que en dicha sociedad ejerce el clima y el entorno físico en donde se asienta, así como la influencia de la presencia de los seres humanos en la transformación de los diferentes espacios y entornos naturales (Domínguez, 2004, p. 42). Es decir, es una disciplina cuyo objetivo es describir y explicar la dimensión espacial de las relaciones entre cultura y naturaleza (Bale, 1999; Domínguez, 2004).

La alfabetización en geografía busca desarrollar: el dominio del lenguaje cartográfico (la escala, la proyección, la simbología, etc.), la capacidad de localización y orientación, el lenguaje numérico presente en los cuadros gráficos y tablas, entre otros (Bale, 1999; Prats, 2002). En términos generales, se debe posicionar como una disciplina, capaz de generar conocimiento útil para la sociedad, lo que implica nuevas formas de alfabetizar (Arenas y Salinas, 2013). Los desafíos apuntan a que desde los primeros años de escolaridad los estudiantes sean capaces de interpretar y analizar los problemas de la sociedad, y entiendan el contexto físico dentro del cual ocurren los conflictos sociales, hasta lograr, comprender las relaciones geográficas en toda su intensidad. Igualmente, el estudiante alfabetizado en geografía, deberá razonar geográficamente abordando, de manera analítica, el espacio desde la experiencia personal y con otros, y a la vez comprometerse con la transformación de aquel (Rodríguez, 2010). Las habilidades comprometidas buscan que el alumno pueda atender a los distintos elementos de este contexto y su complejidad (Haas, 2014) además de aportar a su mayor autonomía.

En concordancia, desde la perspectiva curricular se busca el desarrollo de la conciencia del entorno, la formación del pensamiento histórico, el respeto y valoración de la diversidad humana, la aplicación de los métodos de las ciencias sociales y el pensamiento geográfico. En este sentido, el pensamiento geográfico, se trabaja a lo largo de la educación básica en tres etapas:

a) Identificar y conocer las principales características del espacio, para trabajar la localización, el uso de mapa y otros recursos geográficos.

b) Reconocer que a su alrededor existe un territorio rico en actividades propias del ser humano y el ambiente en el que este se desenvuelve, considerando su impacto en la identidad y cultura nacional.

c) Estudiar el paisaje y el espacio geográfico, desde una perspectiva que integra otras disciplinas, como historia, geografía y ciencias sociales. 
En síntesis, la alfabetización en geografía permitirá al estudiante conocer de manera integral el mundo que lo rodea, para comprender, asumir activa y responsablemente la relación recíproca-bidireccional que se da entre hombre y espacio, entendiendo que este último es el escenario del quehacer humano, tanto en el pasado, presente como futuro.

\section{Metodología}

En el presente artículo se intenta describir las interacciones que propician los docentes en la enseñanza de geografía en un tercero y sexto básico de un establecimiento municipal de la ciudad de Viña del Mar (Chile), a fin de analizar en qué medida dichas interacciones concuerdan con lo que se busca propiciar desde la alfabetización de la disciplina en estudio.

En total se analizaron 11 sesiones de clases correspondientes a dos unidades curriculares. La unidad de tercero básico denominada "Representaciones de la Tierra" contempló 8 sesiones, mientras que la unidad de sexto básico "Riesgos y desastres naturales" consideró 3.

La investigación contempla tres pasos con los que se procede a hacer el análisis de cada caso, a partir de lo que propone Sánchez García y Rosales (2010), como se precisa en la figura 1.

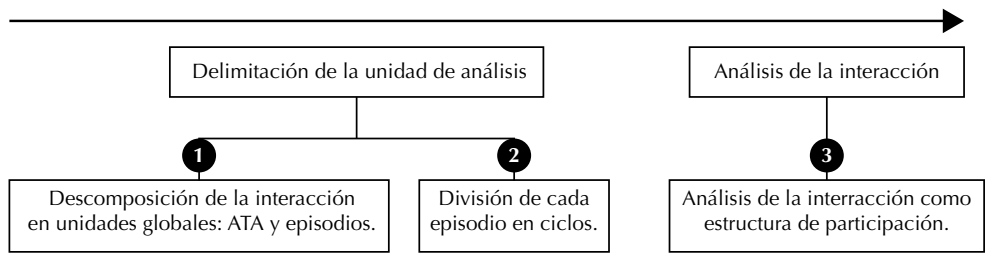

Figura 1. Procedimientos de análisis

Fuente: elaboración propia a partir de Sánchez, García y Rosales (2010).

De esta forma, el primer paso consiste en identificar la unidad de análisis más amplias, en este caso, la unidad curricular. Cada una se segmenta en sesiones, que se dividen en las diferentes actividades típicas de aula (ATA). Es importante señalar que las ATA identificadas fueron definidas desde el estudio realizado por Sánchez y Rosales (2005); mientras que aquellas ATA que no coincidían con lo planteado por el autor fueron definidas a partir de la realidad observada.

Posteriormente, se realizó la segmentación de cada ATA elegida en sus episodios constitutivos. Una vez identificadas las unidades globales (unidad curricular, sesiones, ATA, secuencia de episodios) se establecieron los segmentos más elementales de la interacción de los episodios elegidos, es decir, cada uno de sus ciclos. 
Para identificar un ciclo, lo primero es considerar que estos comienzan con una orden o pregunta inicial y finalizan cuando esta es satisfecha; es decir, cuando se alcanza un acuerdo compartido por los interlocutores. Posteriormente, establecidos los ciclos de cada episodio se inicia el segundo paso del análisis: la identificación de las estructuras de participación, que nos permiten describir cómo se organizan comunicativamente los ciclos de interacción que surgen en cada caso en estudio. La tabla 1 presenta y sintetiza los criterios para la clasificación de las estructuras de participación trabajadas: IRE (iniciación, respuesta, evaluación); IRF (iniciación, respuesta, feedback) e IRF incompleta.

Tabla 1. Criterios para la clasificación de las estructuras de planificación

\begin{tabular}{|ll}
\hline \multicolumn{1}{c}{ Ciclos } & El profesor hace una pregunta pero desiste sin haber \\
frustrados & obtenido una respuesta satisfactoria. \\
& Criterio obligatorio: \\
& -Pregunta que no requiere elaboración (intención evaluativa o recitativa). \\
De manera opcional puede darse adicionalmente \\
alguno de estos elementos: \\
-No hay aproximaciones sucesivas en la respuesta. \\
-Feedback simple (sí/no/bien/mal). \\
Criterio obligatorio: \\
-Pregunta que requiere elaboración (la intención es construir conocimiento). \\
Pero no aparece ninguno de estos elementos: \\
-Aproximaciones sucesivas. \\
incompleta \\
-Feedback complejo. \\
Criterio obligatorio: \\
-Pregunta que requiere elaboración (la intención es construir conocimiento). \\
Debe darse además, alguno de estos elementos: \\
-Aproximaciones sucesivas. \\
-Feedback complejo.
\end{tabular}

Fuente: adaptada de Sánchez, García y Rosales (2010).

\section{Resultados}

Los resultados se organizan considerando las interacciones que propician los docentes en cada unidad curricular en estudio. Cada una será descrita a partir de dos niveles de la práctica pedagógica. En primer lugar, un nivel macro (segmentación de la interacción en unidades de análisis: unidad curricular, ATA, episodios y ciclos) y desde un nivel micro (estructura de participación identificada en los ciclos de interacción: IRE, IRF, IRF Incompleta, monologal, simétricos o ciclo frustrado). 


\section{Estudio de caso 1: Unidad curricular sexto básico "Riesgos y desastres naturales"}

La tabla 2 ilustra cómo se van secuenciando cada una de las unidades de análisis prototípicas de la unidad curricular.

Tabla 2. Despliegue unidades de análisis caso 1: Sexto básico geografía

\begin{tabular}{|c|c|c|c|}
\hline $\begin{array}{l}\text { Unidad } \\
\text { curricular }\end{array}$ & Sesiones & $\begin{array}{l}\text { Actividades } \\
\text { típicas de aula }\end{array}$ & Episodios \\
\hline \multirow{14}{*}{$\begin{array}{l}\text { Riesgos y } \\
\text { desastres } \\
\text { naturales }\end{array}$} & \multirow{14}{*}{ Tres sesiones } & \multirow{4}{*}{$\begin{array}{l}\text { Instrucciones } \\
\text { de la actividad }\end{array}$} & Aclaración de conceptos \\
\hline & & & Instrucción de la actividad \\
\hline & & & Ejemplificación \\
\hline & & & Explicación procedimental \\
\hline & & \multirow{4}{*}{$\begin{array}{l}\text { Resolución } \\
\text { de tareas }\end{array}$} & $\begin{array}{l}\text { Desarrollo de la actividad } \\
\text { con monitoreo }\end{array}$ \\
\hline & & & Aclaración de conceptos \\
\hline & & & Modelado de la actividad \\
\hline & & & Revisión colectiva \\
\hline & & \multirow{6}{*}{ Actividad de cierre } & Valoración personal del contenido \\
\hline & & & Vinculación contenido próxima clase \\
\hline & & & Corrección de las actividades \\
\hline & & & Verificación objetivo de la clase \\
\hline & & & Aclaración de conceptos \\
\hline & & & Resumen \\
\hline
\end{tabular}

Fuente: adaptado de Sánchez, García y Rosales (2010).

Considerando los datos expuestos, podemos constatar que a lo largo de la unidad, tres ATA Ocupan un papel predominante en todas las sesiones. Asimismo, cada una presenta un patrón sistemático de episodios que se repiten en cada sesión.

En primer lugar, en el ATA de instrucciones de la actividad, la docente proporciona una variedad de ejemplos que pretender guiar el trabajo de los estudiantes y anticiparse a las dificultades que se puedan presentar.

A partir de esto, comienza el ATA de resolución de actividades, que es el eje de cuanto acontece. A lo largo de las sesiones, se puede confirmar que la docente propicia instancias de búsqueda de información a través de diferentes recursos de aprendizaje, como el uso de internet y el texto de estudio para responder las preguntas planteadas en una guía de aprendizaje.

Por último, tras la resolución de actividades, se finaliza la clase con el ATA de actividad de cierre. Así, se formalizan los contenidos abordados a partir de actividades como la elaboración de resumen, la corrección de las actividades y la aclaración de conceptos. También, se propician instancias para vincular el contenido visto con experiencias previas de los estudiantes, a fin de favorecer la valorización de lo abordado. 
Respondiendo al análisis de la interacción que surge en el aula a partir del despliegue de las diferentes unidades de análisis, es necesario responder a la forma como se organizan los ciclos de interacción. Para ello, la tabla 3 muestra la estructura de participación predominante en cada ATA prototípica de este estudio de caso.

\section{Tabla 3. Estructura de participación predominante en ATA prototípicas, estudio de caso 1: Riesgos y desastres naturales (sexto básico)}

\begin{tabular}{|c|c|}
\hline $\begin{array}{l}\text { Estructura de participación } \\
\text { (EP) en cada ATA prototípica }\end{array}$ & ATA instrucciones de la actividad con monitoreo \\
\hline EP prototípica & Monologal \\
\hline Ejemplo interacción & $\begin{array}{l}\text { P: Entonces, como para resumir esta parte, un riesgo natural } \\
\text { es una amenaza que sufre nuestro territorio, como esto } \\
\text { es un conjunto de amenazas, pero se transforman en un } \\
\text { desastre cuando las personas se ven afectadas, y no solo } \\
\text { las personas sino que también sus bienes as ciudades, las } \\
\text { poblaciones y también los países. Vamos a conocer hoy día } \\
\text { entonces qué desastres naturales han sido más importantes } \\
\text { a lo largo de la historia, especialmente en el siglo XX; es decir } \\
\text { en el siglo pasado. Bien, empezamos entonces a trabajar. }\end{array}$ \\
\hline Descripción & $\begin{array}{l}\text { Ciclo de interacción que se realiza solamente a partir de } \\
\text { la intervención del docente. Ello implica que el alumno } \\
\text { es un ser pasivo y no se propician oportunidades para } \\
\text { que movilice habilidades, conocimientos disciplinares. }\end{array}$ \\
\hline $\begin{array}{l}\text { Estructura de participación } \\
\text { (EP) en cada ATA prototípica }\end{array}$ & ATA resolución de actividades \\
\hline EP prototípica & IRE \\
\hline \multirow[t]{3}{*}{ Ejemplo interacción } & $\begin{array}{l}\text { P: Ya, por ejemplo, aquí: ¿Cómo se llama eso? Una } \\
\text { placa una encima de la otra. ¿Cómo se llamaba eso? }\end{array}$ \\
\hline & E: En el fondo de la Tierra, eso se llamaba magma. \\
\hline & $\begin{array}{l}\text { P: Bien, porque las placas flotan sobre el magma y se } \\
\text { produce... una de las placas va con la otra haciendo un } \\
\text { movimiento y después una de las placas se monta sobre } \\
\text { otra y ahí se produce, se produce una alteración del magma } \\
\text { porque queda espacio por donde sale el magma. }\end{array}$ \\
\hline Descripción & $\begin{array}{l}\text { La pregunta que abre el ciclo puede responderse solo con } \\
\text { recordar información, no se demanda ningún proceso de } \\
\text { razonamiento, por esta razón, existe una única respuesta. } \\
\text { El feedback del docente es complejo en la medida que } \\
\text { entrega información que complementa la respuesta del } \\
\text { estudiante. Lo mismo que al completarlo le está restando } \\
\text { opciones al alumno de mostrar cuánto comprende al } \\
\text { respecto y si además de recordar, relaciona y argumenta. }\end{array}$ \\
\hline
\end{tabular}




\begin{tabular}{|c|c|}
\hline $\begin{array}{l}\text { Estructura de participación } \\
\text { (EP) en cada ATA prototipica }\end{array}$ & ATA de cierre \\
\hline EP prototípica & IRF \\
\hline \multirow[t]{7}{*}{ Ejemplo Interacción } & $\begin{array}{l}\text { P: ¿Qué impresión les produce a ustedes vivir en un país } \\
\text { tan sísmico que estamos tan expuestos a riesgos? }\end{array}$ \\
\hline & E: La sobrevivencia. \\
\hline & P: ¡Ah!, sobrevivencia, ¿que más? \\
\hline & E: Estar precavidos. \\
\hline & $\begin{array}{l}\text { P: Estar precavidos porque ¿nosotros podemos evitar } \\
\text { la mano de la naturaleza o el poder de la naturaleza? }\end{array}$ \\
\hline & E (todos): No. \\
\hline & $\begin{array}{l}\text { P: No, no podemos ni predecir, ni tampoco podemos } \\
\text { decir a la naturaleza que no haya más riesgos naturales } \\
\text { para nosotros, lo que queda es que nosotros seamos } \\
\text { una población, una comunidad que esté precavida. }\end{array}$ \\
\hline Descripción & $\begin{array}{l}\text { La pregunta que abre el ciclo implica un proceso de } \\
\text { relación entre los contenidos nuevos abordados y las } \\
\text { experiencias y conocimientos previos del estudiantes, } \\
\text { se intenciona que el estudiante genere una reflexión } \\
\text { en torno lo aprendido, de ahí que no puedan ser } \\
\text { respondidas tan solo con recordar información. En } \\
\text { estos ciclos, existen aproximaciones sucesivas que dan } \\
\text { cuenta que no existe una única respuesta correcta. }\end{array}$ \\
\hline
\end{tabular}

Fuente: elaboración propia.

De lo anterior se desprende que las estructuras de participación prototípicas de la unidad son de tipo IRE y monologal, revelando que el conocimiento se construye a partir de preguntas que pueden ser respondidas solo a través del recuerdo de información adquirido durante el desarrollo de la actividad. Asimismo, esta perspectiva vinculada con un tipo de enseñanza tradicional basada en la memorización y repetición de los saberes, da cuenta que el docente es quien interviene en la mayoría del tiempo, planteando preguntas de carácter evaluativo, e incluso, transmitiendo información de manera monologal para aclarar los contenidos abordados, sin propiciar intercambios comunicativos con los estudiantes.

Pese a esto, durante la ATA actividad de cierre, las interacciones se organizan predominantemente a partir de la estructura de participación IRF, lo cual aumenta la participación de los estudiantes. En cuanto al tipo de preguntas planteadas por el docente, estas se enfocan en fomentar permanentemente la reflexión, valorización y vinculación de los contenidos abordados con experiencias previas de los estudiantes.

\section{Estudio de caso 2: Unidad curricular tercero básico "Representaciones de la Tierra"}

La tabla 4 ilustra de forma secuencial el despliegue de cada unidad de análisis correspondientes a este estudio de caso. 
Tabla 4. Despliegue unidades de análisis, estudio de caso 2: tercero básico geografía

\begin{tabular}{|c|c|c|c|}
\hline Unidad curricular & Sesiónes & $\begin{array}{l}\text { Actividades } \\
\text { típicas de aula }\end{array}$ & Episodios \\
\hline \multirow{13}{*}{$\begin{array}{l}\text { Representaciones } \\
\text { de la Tierra }\end{array}$} & \multirow{13}{*}{$\begin{array}{l}\text { Ocho } \\
\text { sesiones }\end{array}$} & \multirow{3}{*}{$\begin{array}{c}\text { Activación } \\
\text { conocimientos } \\
\text { previos }\end{array}$} & Exploración de ideas previas \\
\hline & & & Motivación \\
\hline & & & Exploración de contenidos vistos \\
\hline & & \multirow{7}{*}{$\begin{array}{l}\text { Resolución de } \\
\text { actividades }\end{array}$} & Modelado de la actividad \\
\hline & & & Ubicación espacial \\
\hline & & & $\begin{array}{l}\text { Desarrollo de la actividad } \\
\text { con monitoreo }\end{array}$ \\
\hline & & & Corrección de la actividad \\
\hline & & & $\begin{array}{l}\text { Desarrollo de la actividad } \\
\text { con preguntas guiadas }\end{array}$ \\
\hline & & & Instrucciones de la actividad \\
\hline & & & Aclaración contenido \\
\hline & & \multirow{3}{*}{ Actividad de cierre } & Verificación del contenido. \\
\hline & & & Consolidación del contenido \\
\hline & & & Vinculación próxima clase \\
\hline
\end{tabular}

Fuente: elaboración propia.

De manera específica, la ATA de activación conocimientos previos se lleva a cabo al inicio de cada sesión, cumpliendo diferentes objetivos; mientras que en ocasiones se utiliza para conocer las nociones e ideas previas que poseen los alumnos acerca del contenido a trabajar; en otras sesiones sirve para evaluar el contenido visto en clases anteriores. Por otro lado, la ATA resolución de actividades constituye el eje articulador de la unidad, en tanto, es en estas instancias donde los estudiantes aplican lo aprendido -ya sea trabajando de forma individual, en parejas o grupos-, realizando las actividades y utilizando una gran variedad de herramientas como, videos, programas interactivos (Google Earth), planisferio, mapas y globo terráqueo, que permiten desarrollar las habilidades propias de la disciplina. La ATA de cierre cumple el propósito de verificar el objetivo de clase, para ello el docente suele plantear preguntas a los estudiantes, evidenciando que se hayan comprendido cada uno de los contenidos vistos en la sesión.

Por último, es necesario destacar que antes o durante el desarrollo de cada ATA el profesor dedica tiempo a gestionar y normalizar la clase, ocupando un tiempo prolongado en regular la conducta de los estudiantes con el fin de establecer un ambiente propicio para llevar a cabo las actividades propuestas.

Para dar respuesta a cómo se organizan los ciclos de interacción en esta unidad curricular. La tabla 5 detalla la estructura de participación predominante en cada ATA prototípica de este estudio de caso. 
Tabla 5. Estructura de participación predominante en ATA prototípicas estudio de caso 2: Representaciones de la Tierra (tercero básico básico)

\begin{tabular}{|c|c|c|c|}
\hline $\begin{array}{c}\text { Estructura de } \\
\text { participación (EP) en } \\
\text { cada ATA prototípica }\end{array}$ & $\begin{array}{c}\text { EP } \\
\text { prototipica }\end{array}$ & Ejemplo interacción & Descripción \\
\hline $\begin{array}{c}\text { Activación } \\
\text { conocimientos } \\
\text { previos }\end{array}$ & IRE & $\begin{array}{l}\text { P: ¿Conocen Chiloé? } \\
\text { ¿Han viajado? } \\
\text { E: ¡Síl } \\
\text { E: No. } \\
\text { E: Sí. } \\
\text { P: ¿Sí? ¡Qué bien! } \\
\text { Ahora vamos a saber. }\end{array}$ & $\begin{array}{l}\text { El ciclo se inicia con la } \\
\text { intervención del docente } \\
\text { quien plantea una } \\
\text { pregunta que no requiere } \\
\text { de una elaboración; } \\
\text { más bien busca explorar } \\
\text { los conocimientos } \\
\text { previos de los alumnos, } \\
\text { en donde el docente } \\
\text { entrega un feedback } \\
\text { simple, validando la } \\
\text { respuesta de los alumnos } \\
\text { y motivándolos a } \\
\text { compartir experiencias. }\end{array}$ \\
\hline $\begin{array}{l}\text { Resolución } \\
\text { actividades }\end{array}$ & IRE & $\begin{array}{l}\text { P: Ahora, para ir jugando } \\
\text { un poco, vamos a } \\
\text { ubicar un país vecino } \\
\text { a Chile ¿Cuál es un } \\
\text { país vecino a Chile? } \\
\text { E: Argentina. } \\
\text { P: Argentina, ¿verdad? } \\
\text { P: Redoble de tambores } \\
\text { Jennifer busque el } \\
\text { país vecino de Chile, } \\
\text { que es Argentina } \\
\text { E: Ahí está. } \\
\text { P: Muy bien, ahora el } \\
\text { globo. Fíjense en lo } \\
\text { que está haciendo } \\
\text { la Jennifer, miren. } \\
\text { P: Ahí está, perfecto, } \\
\text { muchas gracias, aplausos. }\end{array}$ & $\begin{array}{l}\text { La pregunta que abre el } \\
\text { ciclo puede responderse } \\
\text { solo con recordar y } \\
\text { observar la información, } \\
\text { no se demanda ningún } \\
\text { proceso de razonamiento, } \\
\text { por esta razón, existe } \\
\text { única respuesta. El } \\
\text { feedback del docente es } \\
\text { simple en la medida que } \\
\text { no se complementa la } \\
\text { respuesta del estudiante. } \\
\text { Desde el punto de vista } \\
\text { de la interacción, solo } \\
\text { se propicia la memoria, } \\
\text { el recordar y no se da } \\
\text { pie a ubicar, localizar } \\
\text { y otras habilidades } \\
\text { que se relacionan con } \\
\text { aquellas que la disciplina } \\
\text { busca alfabetizar. }\end{array}$ \\
\hline Actividad de cierre & Monologal & $\begin{array}{l}\text { P: Chicos, atención. } \\
\text { Recuerden los paralelos; } \\
\text { son muy importantes } \\
\text { para saber dónde está } \\
\text { el norte, el sur, este y el } \\
\text { oeste, estas son líneas } \\
\text { horizontales y la más } \\
\text { importante es la línea } \\
\text { del Ecuador que separa } \\
\text { el hemisferio norte del } \\
\text { hemisferio sur. Ahora } \\
\text { cierren el cuaderno. }\end{array}$ & $\begin{array}{l}\text { Ciclo de interacción } \\
\text { que se realiza a partir } \\
\text { de la intervención del } \\
\text { docente. Los alumnos } \\
\text { no tienen participación } \\
\text { alguna, lo que implica } \\
\text { que el docente los } \\
\text { margina de compartir, } \\
\text { cuestionarse, explicar, } \\
\text { debatir e, incluso, mostrar } \\
\text { valoraciones al respecto. } \\
\text { Desde lo relativo a la } \\
\text { alfabetización en el área. } \\
\text { Esta sería una excelente } \\
\text { instancia para que los } \\
\text { estudiantes evidenciaran } \\
\text { el qué, por qué y para } \\
\text { qué de lo aprendido. }\end{array}$ \\
\hline
\end{tabular}


De manera específica, los intercambios comunicativos prototípicos se organizan a partir de la estructura IRE y Monologal, lo que demuestra que las preguntas realizadas por el docente están orientadas de forma exclusiva a evaluar la adquisición de los contenidos conceptuales abordados en el desarrollo del episodio o consolidar los contenidos trabajados, sin propiciar intercambios comunicativos con los estudiantes, salvo relevando lo meramente memorístico. Pese a lo anterior, las interacciones que se propician en este nivel son, en parte, coherentes con lo propuesto desde el Currículum Nacional, dado que los objetivos en este nivel pretenden desarrollar el pensamiento geográfico desde el reconocimiento de diversos conceptos, categorías y uso de herramientas geográficas. No obstante, si lo que se proyecta es la alfabetización en el área, más que recordar el alumno debiera comprender, relacionar y aplicar los conceptos y para ello otro tipo de interacciones debieran darse, claramente en las que sea más activo. De ahí que recordar los conceptos solo constituye un primer eslabón para la posterior adquisición de habilidades y actitudes propias de la disciplina orientadas a desarrollar una mirada integral y una mejor comprensión de lo que está ocurriendo en el mundo actual.

\section{Conclusiones}

Desde un enfoque sociocultural, cada contexto escolar y de aula implica una determinada realidad, a partir de la cual se construyen significados, formas de ver el mundo, de relacionarse y comunicarse. Lo anterior, para los casos estudiados, se manifiesta dada la pertenencia de la escuela a un contexto social vulnerable y prioritario, con una realidad de grandes necesidades que configura e impacta en lo que los profesores van a privilegiar a nivel del aula. De este modo, ellos, más allá de limitarse a alfabetizar en áreas específicas del saber, deben desarrollar en los alumnos un clima propicio de aula, rutinas de trabajo junto al progresivo desarrollo de conocimientos (conceptual, procedimental y actitudinal) para que en lo venidero las otras capacidades sean posibles de proyectar.

Ahora bien, tanto el tercero básico como el sexto básico configuran realidades distintas en las que los profesores favorecen aprendizajes según las características de los sujetos que aprenden, su nivel de autonomía y las particularidades de cada grupo. Las decisiones sobre qué enseñar y con qué recursos hacerlo dependen de los rasgos contextuales del grupo.

En el ámbito particular de la enseñanza de la geografía, y desde el marco de lo que son las interacciones de aula, se espera que los estudiantes sean capaces de razonar geográficamente, abordando analíticamente el espacio desde la experiencia personal (Rodríguez, 2010), a la luz del tipo y la calidad de interacciones que protagonizan la clase. No obstante, las 
competencias comprometidas requieren de procesos de graduación con diferentes niveles de complejidad, desde los primeros años de escolaridad, comenzando por aprender a ubicarse en el espacio, utilizando diversos conceptos, categorías y recursos, hasta aproximarse a la comprensión de la relación entre los elementos geográficos y la vida del hombre.

En relación con los hallazgos, estos dan cuenta que la interacción en la alfabetización de geografía está guiada por estructuras de participación tradicionales, específicamente IRE. Interacciones que responden a los propósitos de favorecer la atención, el manejo de conceptos, a la vez que motivar la participación de los alumnos, pero centrados en el protagonismo docente. Tal estructura de participación, para el caso del tercero básico, resulta en parte pertinente con lo que las bases curriculares plantean como fines pero solo en una etapa muy inicial y sin perder de vista lo que se espera favorecer con la alfabetización en el área en cuestión. No obstante, en el caso del sexto básico se esperaría una disminución de tal estructura en pro de una mayor participación del alumno, pese a que en algunas ocasiones el docente establece interacciones destinadas a valorar el contenido abordado que en lineamiento con lo curricular y las formas de alfabetizar de la disciplina, cumplen el objetivo de incentivar a los estudiantes hacia una postura más reflexiva y crítica de su propia realidad (Rodríguez, 2010).

Sin embargo, las estructuras de participación predominante (monologal e IRE) manifiestan un carácter evaluativo, reproductivo y centrado en la memorización de saberes, pues tiende a asumir que los conocimientos se construyen a través de preguntas cuyas respuestas requieren tan solo con recordar información o ejecutar una acción que no admite variantes, ni procesos de elaboración, selección o razonamiento (Cazden, 1991), lo cual no favorece el análisis ni el pensamiento crítico esperado en el área.

De esta manera, con los matices de lo que se quiera propiciar contextualmente, este rol del docente limita la participación activa del estudiante, pues las interacciones están orientadas, principalmente, al monitoreo y gestión de la clase. Lo anterior disminuye las posibilidades del alumno de ser desafiado, especialmente en los cursos superiores, hacia posturas más reflexivas y críticas de la realidad estudiada, lo cual es complejo desde la perspectiva en la que con tal omisión no se da cabida a la interpretación y análisis de los problemas de la sociedad en relación de la realidad geográfica. Paralelamente, si bien las interacciones se orientan principalmente a desarrollar contenidos conceptuales, se evidencia intento por enseñar contenidos procedimentales específicos de la disciplina a través de las instrucciones y permanente monitoreo realizado. 
En síntesis, a la luz de estos resultados, se abren oportunidades y desafíos, que relevan la importancia de ir potenciando paulatinamente distintas interacciones que vayan favoreciendo que el alumno crezca en conocimientos, habilidades, actitudes y un pensamiento crítico con el que enfrente y asuma su rol activo como ciudadano del mañana.

\section{Referencias}

Abril, G. (1995). Análisis semiótico del discurso. En J.M. Delgado y J. Gutiérrez (eds.), Métodos y técnicas cualitativas de investigación en ciencias sociales (pp. 210-301). Madrid: Graó.

Álvarez, A. y Del Río, P. (1980). La influencia del entorno en la educación. Infancia y Aprendizaje, 29, 3-33

Álvarez, A., y Del Río, P. (2003). Educación y desarrollo: la teoría de Vygotsky y la zona de desarrollo próximo. En C. Coll, J. Palacios y A. Marchesi (comp.), Desarrollo psicológico y educación. Madrid: Alianza.

Arenas, A., y Araya, F. (noviembre de 2017). Tensions in the Teaching of School Geography in Chile. CGE-UGı Lisbon Symposium: Integrating Knowledge and Understanding in Geography Education. Simposio dirigido por CEMRI Portuguese Open University (Centre of Studies on Migrations and Intercultural Relations), Lisboa, Portugal.

Arenas, A. y Salinas V. (2013). Giros en la Educación Geográfica: renovación de lo geográfico y lo educativo. Revista de Geografía Norte Grande, 56, 143-162.

Bajtín, M. (2002). La estética de la creación verbal. Buenos Aires: Siglo xxı.

Bale, J. (1999). Didáctica de la Geografía en la Escuela Primaria. Madrid: Ediciones Morata.

Benítez, M.L. (1998). Enfoques de psicología cultural: tres aproximaciones al estudio de la relación entre cultura y pensamiento. En M.D. Valiña García y J. Blanco (eds.). I Jornadas de Psicología del Pensamiento (pp. 361-373). Santiago de Compostela: Cursos e Congresos da Universidade de Santiago de Compostela.

Bernstein, B. (1990). Class, codes and control: The structuring of pedagogic discourse. Londres: Routledge.

Cazden, C. (1991). El discurso en el aula. El lenguaje de la enseñanza y del aprendizaje: Barcelona: Paidós.

Centro de Perfeccionamiento, Experimentación e Investigación Pedagógicas, CEIP (2011). La evaluación docente en Chile. Santiago de Chile: Ministerio de Educación de Chile.

Christie, F. (2002). Classroom Discourse Analysis: A functional perspective. Londres: Continuum. 
Coll, C. (2010). Enseñar y aprender en el mundo actual: desafíos y encrucijadas. Pensamiento Iberoamericano, 7, 47-66.

Coulthard, M. (1975). Towards an analysis of discourse: the English used by eachers and pupils. Londres: Oxford University Press.

Cubero, R., Cubero, M., Santamaría, A., Saavedra, Y. y Yossef, J.J. (2005). Aprendizaje y psicología histórico-cultural. Aportaciones de una perspectiva social del aula. Investigación en la Escuela, 62, 5-16.

Domínguez, M. (2004). Didáctica de las Ciencias Sociales para primaria. Madrid: Pearson and Prentice.

Flores, M.P. y Abarzúa, A. (2013). El modelo de evaluación y los instrumentos. En J. Manzi, R. Gonzales y Y. Sun (coord.), La evaluación docente en Chile (pp. 35-62). Santiago de Chile: Centro de Medición de la Pontificia Universidad Católica de Chile

Galton, M., Hargreaves, L., Comber, C. Wall, D. y Pell, A. (1999). Inside the Primary Classroom: 20 Years On. Londres: Routledge.

Haas, V. (2014). Proyecto pedagógico de aula: un ejercicio de empoderamiento profesional. En J.M. Garrido y A. Arenas (coord.), Mejorando las prácticas de evaluación de loos aprendizajes en la docencia universitaria Análisis y experiencias (pp. 297-313). Chile: Ediciones Universitarias de Valparaíso.

Halliday, M.A.K. (1982). Exploraciones sobre las funciones del lenguaje. Barcelona: Editorial Médica y Técnica, S.A.

Halliday, M. y Martin, J. (1993). Writing Science: Literacy and discursive power. Londres: Falmer Press.

Heath, S. (1983). Ways with Words: Language, life, work in communities and classrooms. Nueva York: Cambridge University Press.

Kress, G. (2010). Multimodality: A social semiotic approach to contemporary communication. Londres: Routledge.

Lemke, J. (1998). Multiplying Meaning: Visual and verbal semiotics in scientific text. En J. Martin y R. Veel (eds.), Reading Science: Critical and functional perspectives on discourses of science (pp. 87-113). Londres: Routledge.

Manghi, D. (2013). Representación y comunicación del conocimiento en Educación Media: análisis multimodal del discurso de materiales utilizados para enseñanza escolar de la Historia y de la Historia. Onomázein, 27, 35-52.

Mead, G.H. (1934). Mind, self, and society: from the standpoint of a social behaviorist. Chicago: The University of Chicago Press.

Mehan, H. (1979). Learning lessons. Social organization in the classroom. Cambridge, MA: Harvard University Press.

Moreira, R. (2010). O que é Geografia - nova versão reescrita e atualizada. São Paulo: Brasiliense. 
New London Group (1996). A pedagogy of multi literacies: Designing social futures. Harvard Educational. Review, 66(1), 60-92.

Oteíza, T. (2009). Diálogo entre textos e imágenes: Análisis multimodal de textos escolares desde una perspectiva intertextual. Delta, 25, 657-66.

Prats, J. (2002). Hacia una definición de la investigación en Didáctica de las Ciencias Sociales. Enseñanza de las Ciencias Sociales, 1, 81-89.

Rodríguez, E. (2010). Geografía conceptual: enseñanza y aprendizaje de la geografía en la Educación Básica Secundaria. Colombia: Grupo Geopaideia.

Rogoff, B. (1998). Aprendices del pensamiento: el desarrollo cognitivo en el contexto social. Barcelona: Ediciones Paidós.

Sánchez, E., García, J.L. y Rosales, J. (2010). La lectura en el aula. Qué se hace, qué se debe hacer y qué se puede hacer. Barcelona: Graó.

Sánchez, E. y Rosales, J. (2005). La práctica educativa. Una revisión a partir del estudio de la interacción profesor-alumnos en el aula. Cultura y Educación, 17(2), 147-173.

Vygotsky, L.S. (1988). Pensamiento y lenguaje: teoría del desarrollo cultural de la mente. Barcelona: Paidós.

Wells, G. (2001). Indagación dialógica: hacia una teoría y una práctica socioculturales de la educación. Barcelona: Paidós. 